\title{
Theoretical and experimental study of the effect of fiber heads on the mechanical properties of non-continuous basalt fiber reinforced composites
}

\author{
L. M. Vas $^{1}$, K. Pölöskei ${ }^{1}$, D. Felhös ${ }^{2}$, T. Deák ${ }^{1}$, T. Czigány ${ }^{1,3 *}$ \\ ${ }^{1}$ Department of Polymer Engineering, Faculty of Mechanical Engineering, Budapest University of Technology and \\ Economics, H-1111 Budapest, Múegyetem rkp. 3., Hungary \\ 2Institute of Machine Design, Faculty of Mechanical Engineering, Budapest University of Technology and Economics, \\ H-1111 Budapest, Múegyetem rkp. 3., Hungary \\ ${ }^{3}$ Research Group for Metals Technology, Hungarian Academy of Sciences, H-1111 Budapest, Goldmann tér 3., Hungary
}

Received 27 November 2006; accepted in revised form 26 January 2007

\begin{abstract}
The effect of basalt fibers, produced by the Junkers technology and used as reinforcement in polymer composites, was modeled on the properties of composites, adapting the statistical fiber mat model of Poisson type. The random distribution was approximated by so-called effective spheres that act as defect sites in composites, reducing their strength. The role of fiber heads in strength reduction and the corresponding failure modes were analyzed theoretically using a model and by experiments performed on specimens containing a single fiber head located at different distances from the crack initiation. The applicability of the model was proven both experimentally and by finite element analysis. Based on all these investigations, the effective cross section reduction, and hence the strength reduction (predicted by the model) caused by the presence of fiber heads was proven.
\end{abstract}

Keywords: polymer composites, basalt fiber, modeling and simulation, fiber head, finite element model

\section{Introduction}

Nowadays the most popular reinforcement in polymer composites is glass fiber, which combines high strength and relatively good interfacial adhesion to the polymer matrix with a low price. Recently increasing attention has been paid to fibers of natural origin, among them to basalt fibers $[1,2]$, produced from volcanic basalt rock by melting. Due to its excellent reinforcing properties - both alone and in combination with other reinforcements - it spread rapidly, together with other natural fibers [3, 4]. Cheap, non-continuous basalt fibers produced by the Junkers technology are brittle, which has to be considered when producing composites form them. Simple adaptation of processes used for ther- moplastic or thermosetting matrix composites may result in a significant decrease of the average fiber length and in the deterioration of mechanical properties [5-7]. The essence of the Junkers technology is that the basalt melt flows from the gas fired tank furnace into a fiber-forming device with horizontal axis, consisting of an accelerator cylinder and two fiber-forming cylinders. The fibers formed by centrifugal force are blown away by high pressure air. This way of fiber spinning is especially productive and cheap, but its disadvantage is that smaller or larger 'heads' remain at the end of the fibers.

The aim of this publication is to analyze the influence of these fiber heads formed on basalt fibers produced by the Junkers technology, gaining popu-

*Corresponding author, e-mail: czigany@eik.bme.hu

(C) BME-PT and GTE 
larity as a reinforcement in polymer composites, on the mechanical properties of composites $[8,9]$.

\section{Materials used}

For the experimental tests basalt fibers produced by the TOPLAN Ltd. (Hungary) were used. The matrix material used for polymer/basalt fiber composites (with or without fiber heads), was $\mathrm{H} 384 \mathrm{~F}$ polypropylene produced by TVK Co. (Hungary), while for the single fiber head composites R 359 PP grade was used. The former composite samples were produced by melt mixing and hot pressing at $200^{\circ} \mathrm{C}$, while the latter ones by extrusion at $205^{\circ} \mathrm{C}$ mold temperature followed by cold pressing.

\section{Finite element and analytical modeling, measurement methods}

The presence of fiber heads may affect the mechanical properties of composites in various ways. On the one hand they may improve toughness by anchoring the fiber, and on the other hand they serve as defect sites and reduce the strength properties. Based on our experience, the latter aspect seems to be dominant. First finite element modeling was used to determine the characteristics of the stress zones around the fiber head, and then the consequences of this phenomenon on the strength properties of composites were described by an analytical model. DEN-T specimens manufactured from PP and single fiber head PP sheets were used to determine the model parameters, while the real strength reduction caused by the fiber heads was determined by tensile tests on conventional dumbbell shape specimens. Afterwards the results calculated by the model were compared to the measured ones.

\subsection{Basic experiments needed for the analytical model}

Tensile specimens were manufactured from the pressed composite sheets according to standard ISO 527-1:1993 (E), and tensile strength was calculated from the test carried out on these pieces with a universal tensile tester of Zwick Z020 type, at a tensile rate of $v=2 \mathrm{~mm} / \mathrm{min}$, as suggested by the standard.

The tensile tests of single fiber head composites were carried out on $20 \times 50 \mathrm{~mm}$ size DEN-T speci-
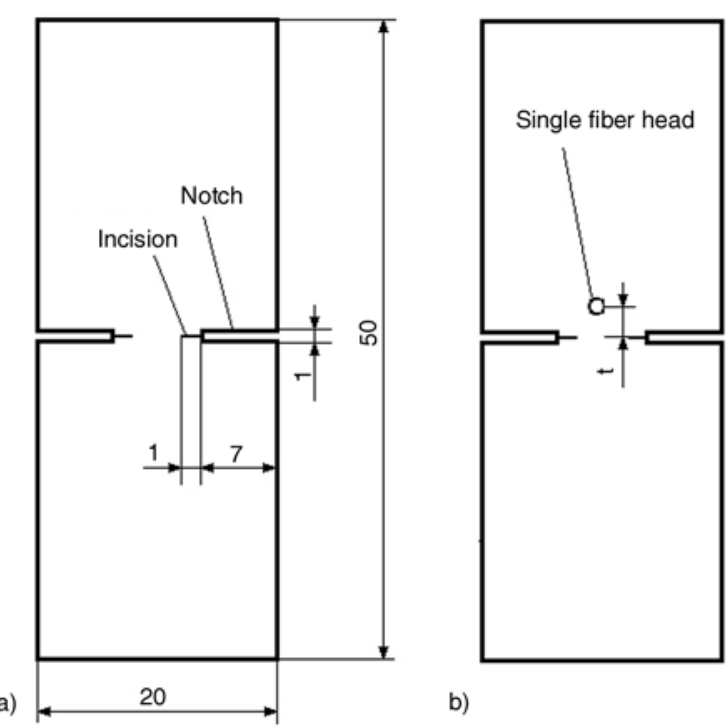

Figure 1. DEN-T specimens notched and cut on both sides, made of unmodified PP (a) and single fiber head PP (b)

mens widely used for measuring EWF (essential work of fracture), machined from $1 \mathrm{~mm}$ thick sheets, and a 1-mm wide and $7 \mathrm{~mm}$ deep notch was prepared on these specimens, later sharpened by a blade. Tensile tests were carried out on a universal tensile tester of Zwick Z005 type at a tensile rate of $v=0.5 \mathrm{~mm} / \mathrm{min}$. The schematic sketch of the specimens can be seen in Figure 1 .

\subsection{Finite element modeling}

Finite element program Cosmos/M was used in our work. An axisymmetric finite element grid made up of Plane2D elements was prepared. The material characteristics were considered constant as a function of temperature. The boundary condition assumed during the analysis was that the bottom plate of the model was fixed in direction $Y$, and the upper plate was loaded with an evenly distributed force. A boundary layer was applied between the fiber heads and the matrix. The material properties of the thin boundary layer between the matrix and the fibre-head were calculated as the averages of the corresponding material properties of the fibre and the matrix material. This thin layer constitutes a transition zone between the rigid fibres and the soft matrix. The nodes of the finite element mesh at the border of the different materials are common, so the properties of these different materials are matched with each other. The model was cooled down from $160^{\circ} \mathrm{C}$ to $25^{\circ} \mathrm{C}$ in one step. Force con- 


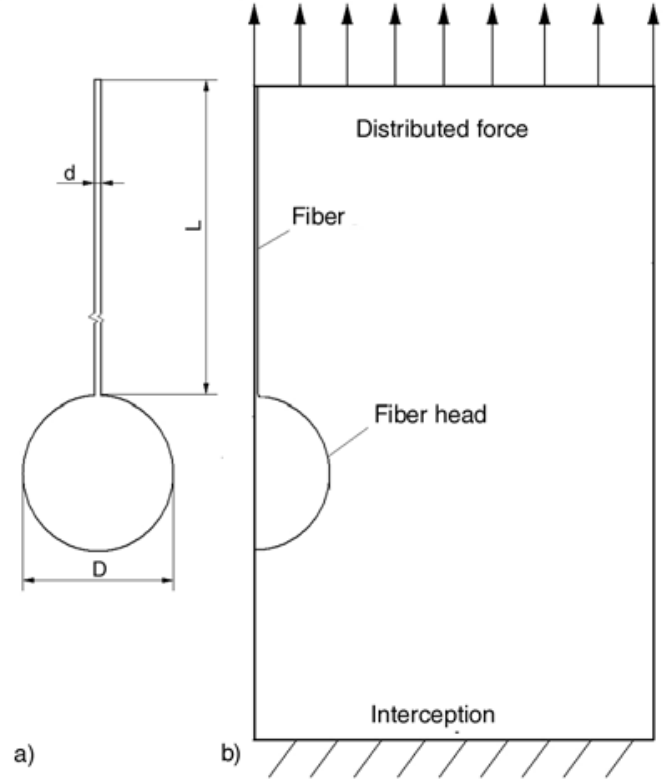

Figure 2. Approximation of the applied fiber head with a sphere (a), and the schematic sketch of the axisymmetrical finite element model (b)

trol and Newton-Raphson method were used in the simulation of the tensile process.

The characteristic size of fiber heads is in the order of tens to hundreds of $\mu \mathrm{m}$, their shape is ellipsoidal or sometimes conical, but they can be well approximated by an equivalent sphere. Figure 2 a shows the model of a spherical fiber head, while Figure 2b includes the schematic sketch of the axisymmetric finite element model.

Table 1. The values of the geometrical parameters of fiber heads

\begin{tabular}{|l|c|c|c|}
\hline \multicolumn{1}{|c|}{ Fiber head shape } & $\mathbf{d}[\boldsymbol{\mu m}]$ & $\mathbf{D}[\boldsymbol{\mu m}]$ & $\mathbf{L}[\mathbf{m m}]$ \\
\hline Sphere & 10 & 100 & 1 \\
\hline Half sphere + truncated cone & 10 & 100 & 1 \\
\hline
\end{tabular}

Table 2. The mechanical properties of materials used in the finite element model

\begin{tabular}{|l|c|c|c|}
\hline & PP & Interface & Basalt fiber \\
\hline Tensile strength [MPa] & 30 & 315 & 600 \\
\hline Yield strength [MPa] & 22 & 311 & - \\
\hline Tensile mod. of elast [GPa] & 1 & 40.5 & 80 \\
\hline
\end{tabular}

Table 3. The thermal properties of materials used in the finite element model

\begin{tabular}{|l|c|c|c|}
\hline & PP & Interface & Basalt fiber \\
\hline $\begin{array}{c}\text { Coefficient of thermal } \\
\text { conductivity }[\mathrm{W} /(\mathrm{mK})]\end{array}$ & 0.22 & 0.51 & 0.8 \\
\hline Specific heat $[\mathrm{kJ} /(\mathrm{kgK})]$ & 1.7 & 1.27 & 0.84 \\
\hline Density $\left[\mathrm{kg} / \mathrm{m}^{3}\right]$ & 910 & 1705 & 2500 \\
\hline $\begin{array}{c}\text { Linear coefficient of ther- } \\
\text { mal expansion }[1 / \mathrm{K}]\end{array}$ & $1.8 \cdot 10^{-5}$ & $1.25 \cdot 10^{-5}$ & $7 \cdot 10^{-6}$ \\
\hline
\end{tabular}

Table 1 includes the values of the geometrical parameters shown in Figure 2.

The strength data of the fiber and the matrix based on the literature are shown in Table $2[4,10]$.

The values used in the modeling of thermal effects are listed in Table 3 [11].

\subsection{Analytical model}

Our aim was to model the random distribution of fiber heads and the random fiber head sizes, as well as their stress concentration zones. The fiber heads are approximated by equivalent spheres having the same volume as those of the fiber heads. The socalled 'effect zones' - drawn around the equivalent spheres -, are considered as $100 \%$ defect sites. The effect zones include the fiber head, the weak adhesion sites between the fiber head and the matrix, as well as the stress concentration zone around the fiber head (Figure 3.). The volume ratio between the fiber heads and the other parts of the composite (containing no such kind of defect site) is assumed to determine the decrease in strength.

In order to handle this problem, a statistical fiber mat model of Poisson type [12, 13] used also for describing the so called fiber flows [12-16] was adapted by substituting the fibers by spheres where a sphere $G(r, P)(1)$ was defined as a spherical volume around point $P$ with $r$ radius in the three dimensional real space $\left(R^{3}\right)$ :

$G(r, P)=\left\{Q \in R^{3}: d(P, Q) \leq r\right\}$

where $d(P, Q)$ is the Euclidean distance of $P\left(x_{P}, y_{P}, z_{P}\right)$ and $Q\left(x_{Q}, y_{Q}, z_{Q}\right)(2)$ :

$$
d(P, Q)=\sqrt{\left(x_{Q}-x_{P}\right)^{2}+\left(y_{Q}-y_{P}\right)^{2}+\left(z_{Q}-z_{P}\right)^{2}}
$$
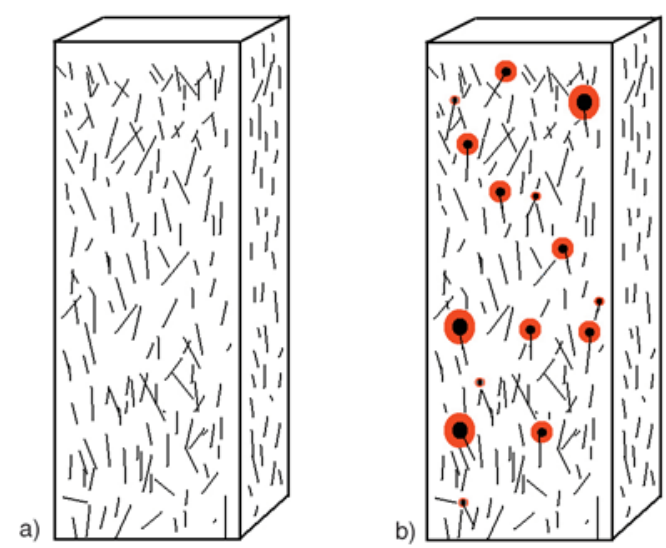

Figure 3. Short fiber reinforced composite body without fiber heads (a) and with fiber heads (b) 
Accordingly, the fiber heads of different shapes and $V_{0}$ volume can be substituted by equivalent spheres of identical volume. The radius of this sphere, $\rho_{0}$ (3) is the following:

$\rho_{0}=\sqrt[3]{\frac{3 V_{0}}{4 \pi}}$

$\rho_{0}$ is a stochastic variable, the distribution function of which is $Q_{\rho_{0}}(r)$, with finite expected value and standard deviation $\left(E \rho_{0}=\bar{\rho}_{0}, D \rho_{0}=\sigma_{\rho_{0}}\right)$. The centre of the equivalent sphere is point $C$, it can be described by $G_{0}=G\left(\rho_{0}, C\right)$.

The so called 'effect zone' around the equivalent sphere can be described by $G(\rho, C)(4)$ (radius: $\rho>\rho_{0}$ ), and is defined as a volume, wherein the stress is significantly larger than in the other parts of the composite. This spherical shell of thickness $r_{e}$ around the equivalent sphere can be expressed by a sphere of radius $\rho$ (Figure 4).

$G(\rho, C)=G\left(r_{e}, G_{0}\right)=G\left(r_{e}, G\left(\rho_{0}, C\right)\right)=\bigcup_{P \in G_{0}} G\left(r_{e}, P\right)$

In general the radius of the effect zone around the effective sphere depends on the radius of the equivalent sphere (5):

$\rho=\rho_{0}+r_{e}\left(\rho_{0}\right)$

If simple special cases are considered, $r_{e}$ may be constant and independent of $\rho_{0}$ or may be proportional to it. In the latter case their ratio can be characterized by a constant $\left(\alpha_{e}\right)(6)$ :

$r_{e}=\alpha_{e} \rho_{0}$

the resultant radius (7):

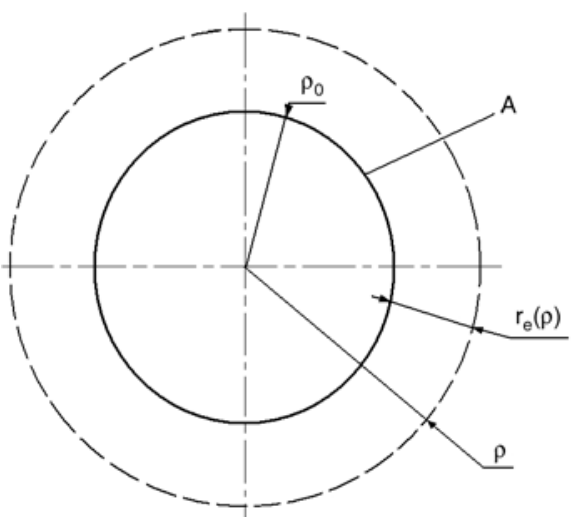

Figure 4. Effective sphere equivalent with a fiber head

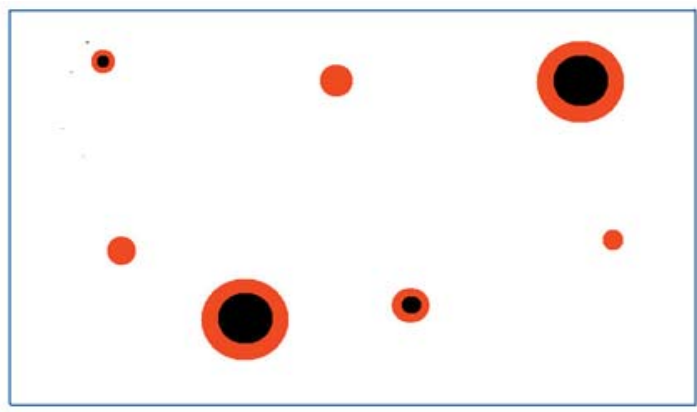

Figure 5. Spheres equivalent with fiber heads and their surrounding effective spheres in the cross section of the composite

$\rho=\left(1+\alpha_{e}\right) \rho_{0}=a_{e} \rho_{0}$

where $a_{e}(8)$ is a constant and based on Equation (7):

$a_{e}=1+\alpha_{e}$

The distribution of these effect zones (and that of the equivalent speheres) in a composite is random (Figure 5). Consequently, the distribution of the effective spheres can be described by a random sphere set (a set of defect sites or heterogeneities), in which the center point of the sphere is an element of a random point set (named point process in the theory of stochastic processes [13]) and its radius is a random variable.

It has been proven by several experiments that the distribution of different flaws in various materials can be described by the Poisson distribution [13, 17]. Based on this fact - assuming that the spatial distribution of spheres is homogeneous - the centers of these spheres can be characterized by a Poisson point process $\left(X^{*}\right)[12,13]$ in the three dimensional real space. If $B \subset R^{3}$ is a closed convex set, and the number of sphere center points that fall into $B$ is $\chi_{B}=\#\left(B \cap X^{*}\right)$, then the probability that $\chi_{B}$ is exactly $k=1,2, \ldots, \infty$ can be given by a Poisson distribution according to (9).

$$
P\left(\chi_{B}=k\right)=\frac{\left(K V_{B}\right)^{k}}{k !} \mathrm{e}^{-K V_{B}}
$$

where $K$ is the density of center points and $V_{B}=$ $V(B)$ is the volume of $B$. Consequently, the expected value and square deviation of $\chi_{B}$ in this case can be obtained according to (10):

$E \chi_{B}=K V_{B}=D^{2} \chi_{B}$ 


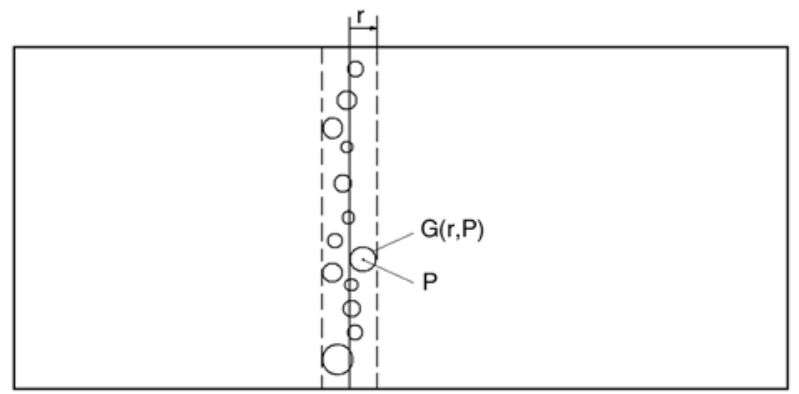

Figure 6. Effect zone of cross section B and the effective spheres within

Since the size of the fiber heads is independent of their position, (o is a variable independent from $X^{*}$ and consequently from $\chi_{B}$.

The fiber heads serve as defect sites in the composite, hence the effective cross section decreases, and the risk of failure increases. Let $B \subset R^{3}$ be a cross section of the composite body. In this case $B$ is a two dimensional rectangle. The intersection of the effective spheres with this cross section can be seen in Figure 6.

If the properties of the number of intersections $\left(\xi_{B}\right)$ of the spheres and $B$ are analyzed, the distribution of $\xi_{B}$ can be determined. Obviously, the spherical vicinity of point $C$ of radius $r$ intersects $B$ if $C$ falls within the r radius $G(r, B)$ spherical vicinity of $B$ $[12,13]$. This event of intersection can be characterized by relation (11):

$$
\{B \cap G(r, C) \neq \varnothing\}=\{C \in G(r, B)\}
$$

where the vicinity of $B$ within the specimen (the intersection of the $r$ radius vicinity of $B$ and the specimen) is a prism of base $A$ and width of $2 r$.

It can be proven that $\xi_{B}$ is of Poisson distribution with the parameters expressed by (12):

$N_{B}=E\left(\xi_{B}\right)=E[K V(G(\rho, B))]=K E[2 A \rho]=2 K A E(\rho)$

since the volume of the vicinity of $B$ in the specimen is $2 A \rho$. This way the expected effective volume can be given by the expected value of the effective volume of $B$ according to (13).

$V_{B E}=E[V(G(\rho, B))]=2 A E(\rho)$

The total volume of spheres intersecting $B$ is given by (14):

$\Phi_{B e}=\sum_{i=1}^{\xi_{B}} V\left(G\left(\rho_{i}, C_{i}\right)\right)=\frac{4}{3} \pi \sum_{i=1}^{\xi_{B}} \rho_{i}^{3}$
Equation (14) is a sum containing a random number of terms where $\xi_{B}$ and $\rho$ are not independent of each other since the vicinity of $B-$ once $\xi_{B}$ is known - depends on $\rho$ (15).

$$
E\left(\Phi_{B e}\right)=E\left[E\left(\Phi_{B e} \mid \rho\right)\right]=\frac{4}{3} \pi E\left[E\left(\sum_{i=1}^{\xi_{B}} \rho_{i}^{3} \mid \rho\right)\right]
$$

The conditional expected value [13] in (15) is valid for a fixed $\rho$ if $\rho_{i}=\rho$ and $\xi_{B}$ is the number of intersections of similar spheres with $B$. When calculating this conditional expected value, Eqution (16) is obtained:

$$
E\left(\sum_{i=1}^{\xi_{B}} \rho_{i}^{3} \mid \rho\right)=E\left(\xi_{B} \mid \rho\right) \rho^{3}=K A 2 \rho \cdot \rho^{3}=2 K A \rho^{4}
$$

wherein Equation (13) was also used. With the help of relation (16), the expected value can be calculated based on (15), according to (17):

$$
V_{B e}=E\left(\Phi_{B e}\right)=\frac{8}{3} \pi K A E\left(\rho^{4}\right)
$$

Knowing $V_{B e}$, the volume ratio that compares the volume outside the effective sphere to the total inspected volume (18) can be determined:

$$
\begin{aligned}
& 0<\Phi_{B}=\frac{V_{B E}-V_{B e}}{V_{B E}}=1-\frac{8 \pi K A E\left(\rho^{4}\right)}{3 \cdot 2 A E(\rho)}= \\
& 1-\frac{4 \pi K}{3} \frac{E\left(\rho^{4}\right)}{E(\rho)}<1
\end{aligned}
$$

Based on the results, the fraction of the residual intact area of $B$ can be determined, and this way the extent of strength reduction can be estimated.

Another possibility to characterize the effect of fiber heads is to determine the volume sum of all the effective spheres in the effect zone of cross section $B$, the radius of which is $\bar{\rho}=E(\rho)$. The number of these spheres can be estimated by (19) from the average number of the center points that fall into the effect zone of $B$ :

$$
N_{B V}=E\left(\chi_{G(\bar{\rho}, B)}\right)=K E(2 A \bar{\rho})=2 K A E(\rho)
$$

From Equations (12) and (19) it is visible that $N_{B V}=N_{B}$, that is the number of spheres intersecting $B$ (type IS) is equal to that of spheres the center point of which falls into the effect zone of $B$ (type $\mathrm{CF}$ ). Let the set of sphere center points of those spheres, which do not intersect $B$ be NIS and the set 


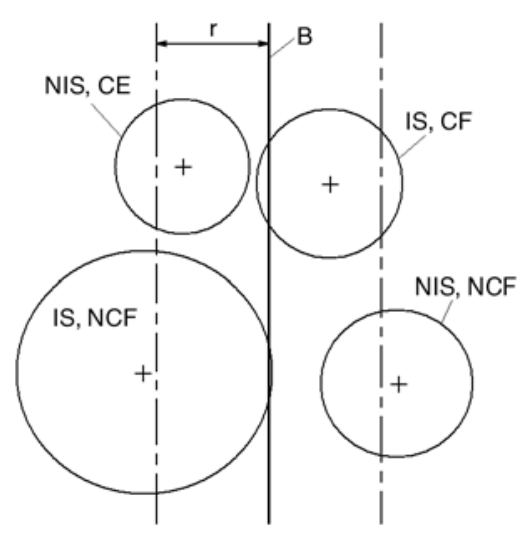

Figure 7. Relative position of center point $C$ of effective spheres relative to the $\mathrm{r}$ radius vicinity of cross section $B$

of center points that do not fall into the effect zone $B$ be NCF (Figure 7).

Let $V_{B V}$ be the expected value of the volume sum in question that can be calculated by the following sum with a constant number of terms (20):

$$
\begin{aligned}
V_{B V}= & E\left[\sum_{i=1}^{N_{B V}} \frac{4 \pi \rho^{3}}{3}\right]=\sum_{i=1}^{N_{B V}} \frac{4 \pi \rho_{i}^{3}}{3}=N_{B} \frac{4 \pi}{3} E\left(\rho^{3}\right)= \\
& \frac{8}{3} \pi K A E(\rho) E\left(\rho^{3}\right)
\end{aligned}
$$

A ratio (21) - similar to (18) - can also be determined using Equation (17):

$$
\begin{aligned}
& \Phi_{B V}= \frac{V_{B E}-V_{B V}}{V_{B E}}=1-\frac{8 \pi K A E(\rho) E\left(\rho^{3}\right)}{3 \cdot 2 A E(\rho)}= \\
& 1-\frac{4 \pi K}{3} E\left(\rho^{3}\right)
\end{aligned}
$$

$N_{B}$ contains effective spheres of type (IS, CF) as well as spheres of (IS, NCF) type, while $N_{B V}$ contains (IS, CF) and (NIS, CF) types, considering an average radius vicinity of $B$. Consequently, the expected values of the numbers of types (IS, NCF) and (NIS, CF) are the same.

The ratio between the volume of spheres that intersect $B\left(V_{B e}\right)$ and the volume of those that fall into the effect zone of $B\left(V_{B V}\right)$ can be determined based on (22):

$\frac{V_{B e}}{V_{B V}}=\frac{E\left(\rho^{4}\right)}{E(\rho) E\left(\rho^{3}\right)}$

Hence, in case of $\rho>0$, the ratio becomes larger than 1 according to Equation (22). The reason for this is that the IS type spheres that are not of type
CF (IS, NCF) are larger than those of CF type but do not belong to type IS (NIS, CF). Consequently, $V_{B e}$ gives a better base to model the effect of fiber heads on the composite behavior.

Knowing the values of $V_{B e}$ the effective cross section area $\left(A_{\text {eff }}\right)$ can be estimated (23) supposing that the thickness of the effect zone remains $2 E(\rho)$ :

$$
A_{\text {eff }}=\frac{V_{B E}-V_{B e}}{2 E(\rho)}=\frac{2 A E(\rho)-\frac{8}{3} \pi K A E\left(\rho^{4}\right)}{2 E(\rho)}=A \Phi_{B}
$$

This equation remains valid if $\rho$ is substituted by $\rho_{0}$.

The main dimensions of the geometrical shapes of the fiber heads found in the basalt fiber set produced under 'normal' operation conditions were determined, and this way their volume could be calculated, and this distribution is shown in Figure 8. It can be seen that frequency decreases steeply as the volume increases; this behavior can be approximated by an exponential curve that can be described by the general formula of Equation (24):

$y=\frac{1}{\lambda} e^{-\frac{V_{0}}{\lambda}}$

where $V_{0}$ is the fiber head volume and $\lambda=$ $17.94 \cdot 10^{-3} \mathrm{~mm}^{3}$.

Since the distribution function of the volume of fiber heads - consequently the volume of the equivalent spheres - is of exponential characteristic [13, 17], its parameter, $\lambda$ is the reciprocal of the average

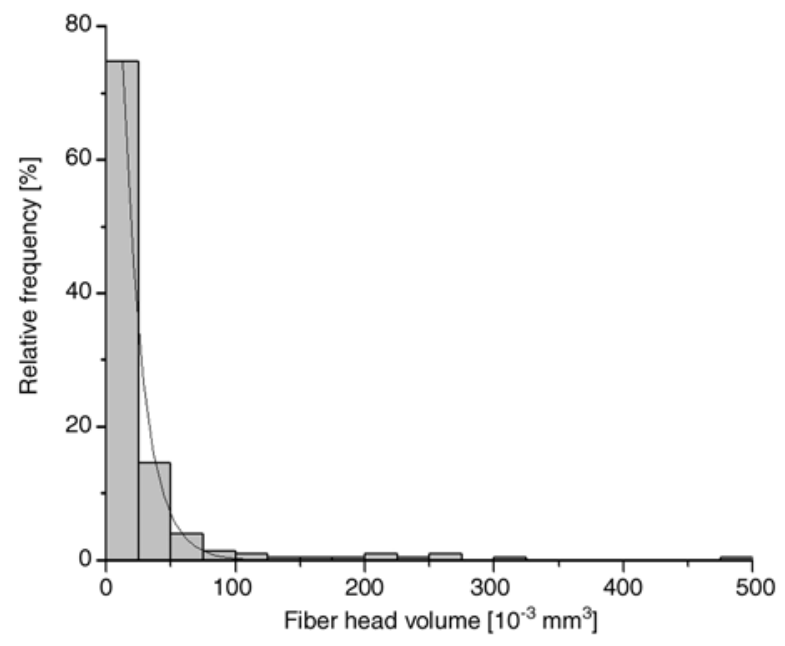

Figure 8. Characteristic fiber head volume distribution of basalt fibers (based on 226 samples) 
volume of the fiber heads. Accordingly, using Equations (3), (12) and (17) the volume of equivalent spheres can be calculated easily. With the help of Equation (3), the equivalent radius $\left(\rho_{0}\right)(25)$ can be calculated using the fiber head distribution found to be exponential:

$E\left(\rho_{0}^{n}\right)=\int_{0}^{\infty} r^{n} \mathrm{~d} Q_{\rho_{0}}(r)=\int_{0}^{\infty}\left(\frac{3 x}{4 \pi}\right)^{\frac{n}{3}} \mathrm{~d} Q_{V_{0}}(x)=$

$\int_{0}^{\infty}\left(\frac{3 x}{4 \pi}\right)^{\frac{n}{3}} \lambda \mathrm{e}^{-\lambda x} \mathrm{~d} x=\left(\frac{3}{4 \pi \lambda}\right)^{\frac{n}{3}} \int_{0}^{\infty} z^{\frac{n}{3}} \mathrm{e}^{-z} \mathrm{~d} z=$

$\left(\frac{3}{4 \pi \lambda}\right)^{\frac{n}{3}} \Gamma\left(\frac{n}{3}+1\right)$

where $\Gamma(p)$ is the gamma function [13,17] $(p>0$ real value), the values of which in case $n=1,3$ and 4 are $\Gamma(4 / 3)=0.89298, \Gamma(2)=1$ and $\Gamma(7 / 3)=$ 1.19069 , respectively. On the basis of these results, the quantities according to Equations (17) and (21) can be rewritten in the following way ((26), (27) and (28)):

$\frac{V_{B e_{0}}}{V_{B S_{0}}}=\frac{E\left(\rho_{0}^{4}\right)}{E\left(\rho_{0}\right) E\left(\rho_{0}^{3}\right)}=\frac{\Gamma(7 / 3)}{\Gamma(4 / 3)}=1.3333=\frac{4}{3}$

$\Phi_{B_{0}}=1-\frac{4 \pi K}{3} \frac{E\left(\rho_{0}^{4}\right)}{E\left(\rho_{0}\right)}=1-\frac{K}{\lambda} \frac{\Gamma(7 / 3)}{\Gamma(4 / 3)}=1-\frac{4}{3} \frac{K}{\lambda}$

$\Phi_{B_{0}}=1-\frac{4 \pi K}{3} E\left(\rho_{0}^{3}\right)=1-\frac{K}{\lambda}$

Consequently, the volume of spheres intersecting $B$ is 1.33 times larger than that of spheres with centers falling within the effect zone of $B$. The former method was used in our calculations. Since $\Phi_{B_{0}}<1$, the relation is valid if $K<3 \lambda / 4$. This way the fourth moment of the radius $(\rho)$ of the effective spheres (29) corresponding to (25) can be calculated by Equation (5) or (7). Using Equation (5) leads to a complicated expression:

$$
\begin{gathered}
E\left(\rho^{n}\right)=\int_{0}^{\infty} r^{n} \mathrm{~d} Q_{\rho}(r)=\int_{0}^{\infty} r^{n} \mathrm{~d} Q_{\rho_{0}}\left(r-r_{e}\right)= \\
\int_{0}^{\infty}\left(r_{0}+r_{e}\right)^{n} \mathrm{~d} Q_{\rho_{0}}\left(r_{0}\right)
\end{gathered}
$$

In case of Equation (7) the result differs from Equation (25) only in a constant factor according to (30):

$$
\begin{aligned}
& E\left(\rho^{n}\right)=\int_{0}^{\infty} r^{n} \mathrm{~d} Q_{\rho}(r)=\int_{0}^{\infty} r^{n} \mathrm{~d} Q_{\rho_{0}}\left(\frac{r}{a_{e}}\right)= \\
& a_{e}^{n} \int_{0}^{\infty} r_{0}^{n} \mathrm{~d} Q_{\rho_{0}}\left(r_{0}\right)=a_{e}^{n} \int_{0}^{\infty}\left(\frac{3 x}{4 \pi}\right)^{\frac{n}{3}} \mathrm{~d} Q_{V_{0}}(x)= \\
& a_{e}^{n} \int_{0}^{\infty}\left(\frac{3 x}{4 \pi}\right)^{\frac{n}{3}} \lambda \mathrm{e}^{-\lambda x} \mathrm{~d} x=a_{e}^{n}\left(\frac{3}{4 \pi \lambda}\right)^{\frac{n}{3}} \int_{0}^{\infty} z^{\frac{n}{3}} \mathrm{e}^{-z} \mathrm{~d} z= \\
& a_{e}^{n}\left(\frac{3}{4 \pi \lambda}\right)^{\frac{n}{3}} \Gamma\left(\frac{n}{3}+1\right)
\end{aligned}
$$

Using these formulae, the volume ratios according to (18), (21) and (22) as well as the effective cross section (23) can be calculated.

\section{Results and discussion}

\subsection{Finite element results}

The tensile test of basalt fibers with fiber heads embedded into PP matrix was modeled in the finite element simulations. Simple tensile tests were carried out on the model, and calculations were completed in order to determine the thermal stresses that arise when the unloaded model is cooled down from $160^{\circ} \mathrm{C}$ to $25^{\circ} \mathrm{C}$, and finally the tensile test of the model loaded with thermal stresses was simulated. Figure 9 shows the results of the finite element simulation of the simple tensile test, including the von Mises type equivalent stress and equivalent strain that arise if an interface is applied in the vicinity of the fiber head.

Figure 9a reveals that the stress zones are elongated in the direction of loading $(Y)$ and have a shape of a deformed ellipse. The stress zone below the fiber head is more significant than the one above it. Based on all these it can be stated that the separation of the fiber head/matrix is likely, hence the additional stresses in the direction of loading are significant, and have to be taken into consideration during strength dimensioning. The equivalent strain introduced in Figure $9 \mathrm{~b}$ reaches the peak value at the border of the fiber head.

Figure 10a shows the calculated results of the finite element simulation of cooling down from $160^{\circ} \mathrm{C}$ to $25^{\circ} \mathrm{C}$. The detailed part reveals that additional stress arises evenly around the fiber head. This stress is large close to the surface of the fiber head but decreases quickly if the distance increases. The 

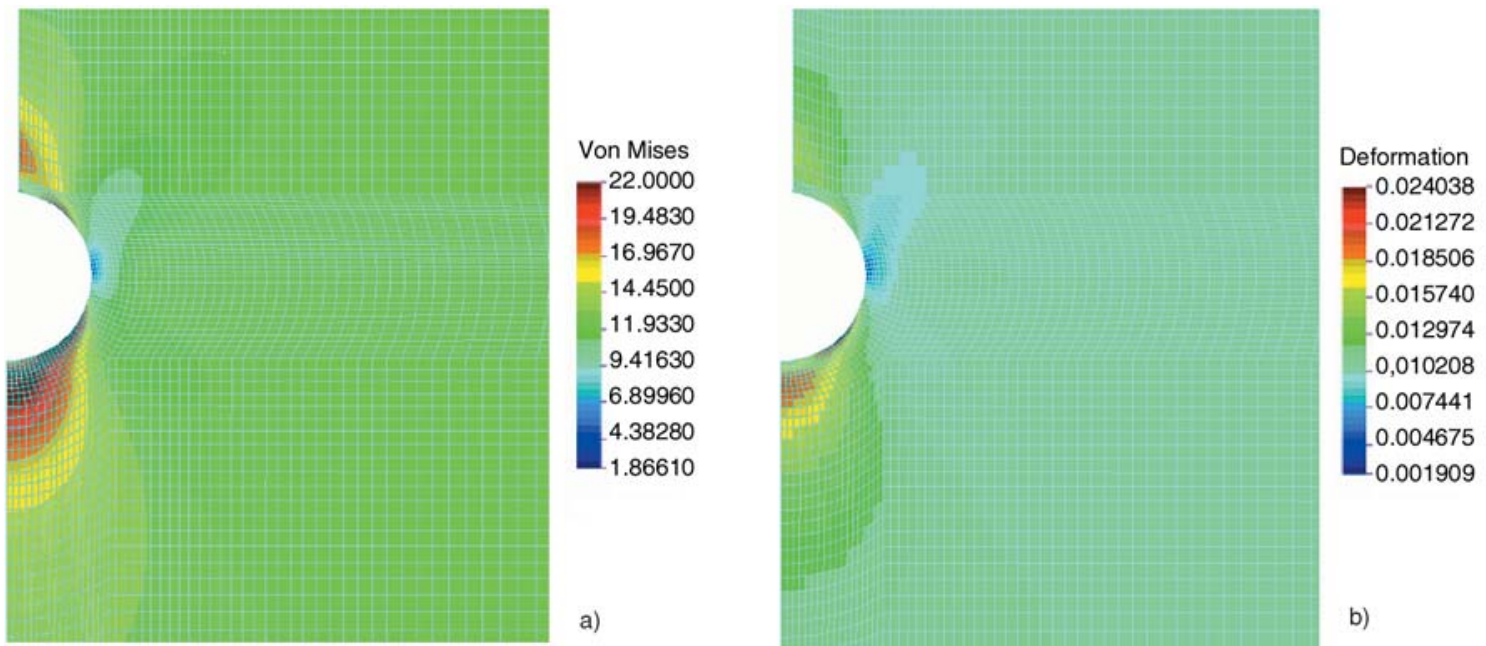

Figure 9. In case of spherical fiber head evolving von Mises stress (a) and equivalent strain (b) in the vicinity of the fiber head when interface owing to tensile loading $\left(F_{e}=11.6 \mathrm{~N}\right)$ is applied
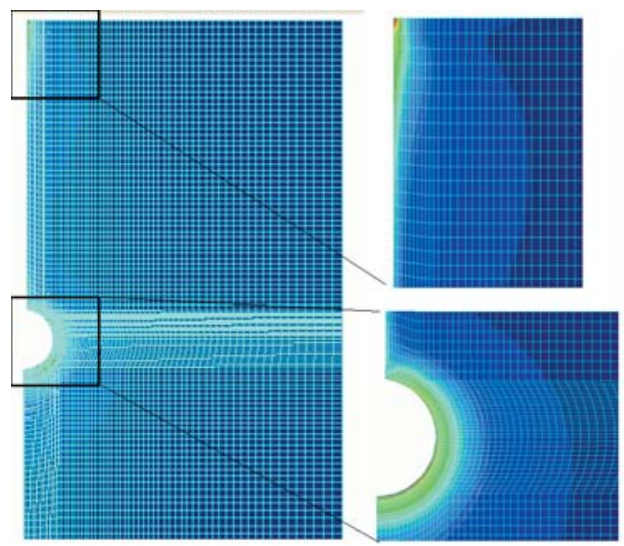

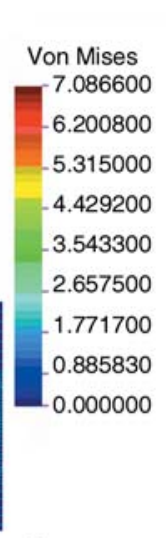

a)

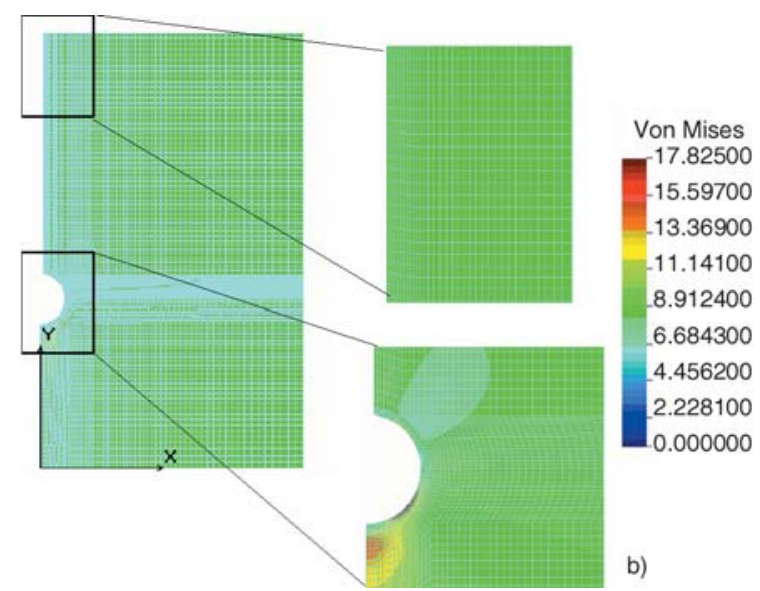

Figure 10. The effect of shrinkage stress (a), and the simultaneous effect of tensile loading $\left(F_{e}=11.6 \mathrm{~N}\right)$ and shrinkage stress during cooling down from $160^{\circ} \mathrm{C}$ to $25^{\circ} \mathrm{C}$ (b) in case of spherical fiber heads

effect zone is much smaller than that experienced in case of tensile strength. Accordingly, it is expected to be of greater importance in case of interfacial layer separation and the yielding of the matrix material around the fiber head.

Figure $10 \mathrm{~b}$ illustrates the calculated finite element results of the combined stress of cooling down from $160^{\circ} \mathrm{C}$ to $25^{\circ} \mathrm{C}$ and the simple loading. These values differ from the stress distribution that forms on samples exposed to tensile tests without cooling. The stresses around the fiber head embedded in PP that arise due to the increased tensile loading can be found in Figure 11. The tensile strength is increased to a three times higher value in order to be able to study the yielding phenomenon of the matrix material around the fiber head. The yielding zones can be observed well at this level of loading. Based on Figure 11 it can be stated that the stress map obtained agrees well with the data found in the

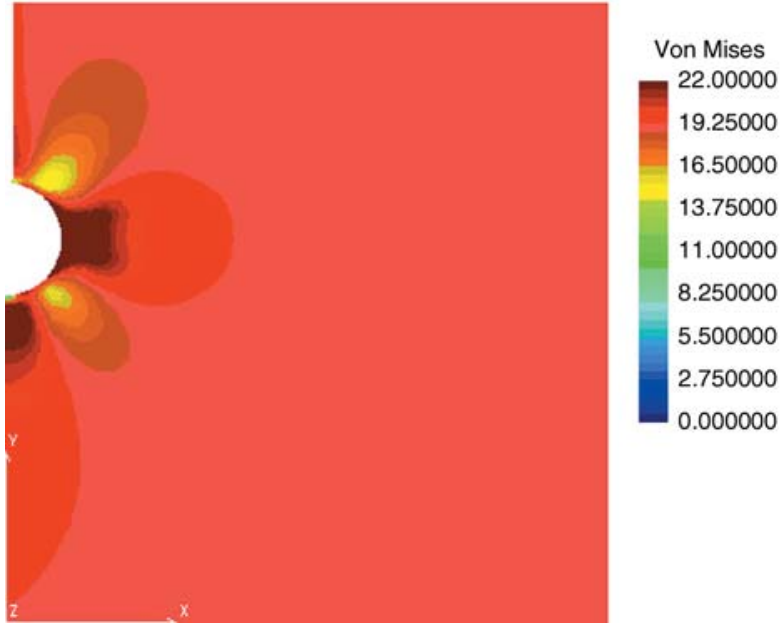

Figure 11. The simultaneous effect of tensile loading $\left(F_{e}=34.8 \mathrm{~N}\right)$ and shrinkage stress during cooling down from $160^{\circ} \mathrm{C}$ to $25^{\circ} \mathrm{C}$ in the non-linear loading stage in case of spherical fiber heads 
literature [18]. Since the yield point of the matrix is $22 \mathrm{MPa}$, the von Mises stress does not exceed this value if loading is increased. However, the size of yielded areas increases if loading is increased. This larger area characterizes the yielding zones. The largest yielding occurred at the meridian of the fiber head due to the changed interfacial relations, and this differs from that Figure 9 shows. The size of the stress concentration zone, oriented in the direction of the fiber, decreased significantly.

\subsection{Experimental determination of the parameters of the analytical model}

The verification of the processes and their parameters is essential before the described theoretical model can be applied. Hence specimens containing PP matrix, single fibers and single fiber heads were manufactured, i.e. pressed from an extruded melt. Single fiber and single fiber head composite tensile tests were carried out on these specimens.

In case of the pressed samples it has been found that the results for single fiber head differed from the PP type R359 in the shape of the tensile curve and in the failure mode as a function of the $t$ fiber head distance defined in Figure 1b. If $\mathrm{t}$ was small enough (ca. $<0.5 \mathrm{~mm}$ ), the line of the crack diverged from the straight line that connects the two notches, and went through the fiber head (Figures 12a and b). If the distance was somewhat larger, the line of the crack did not go through the
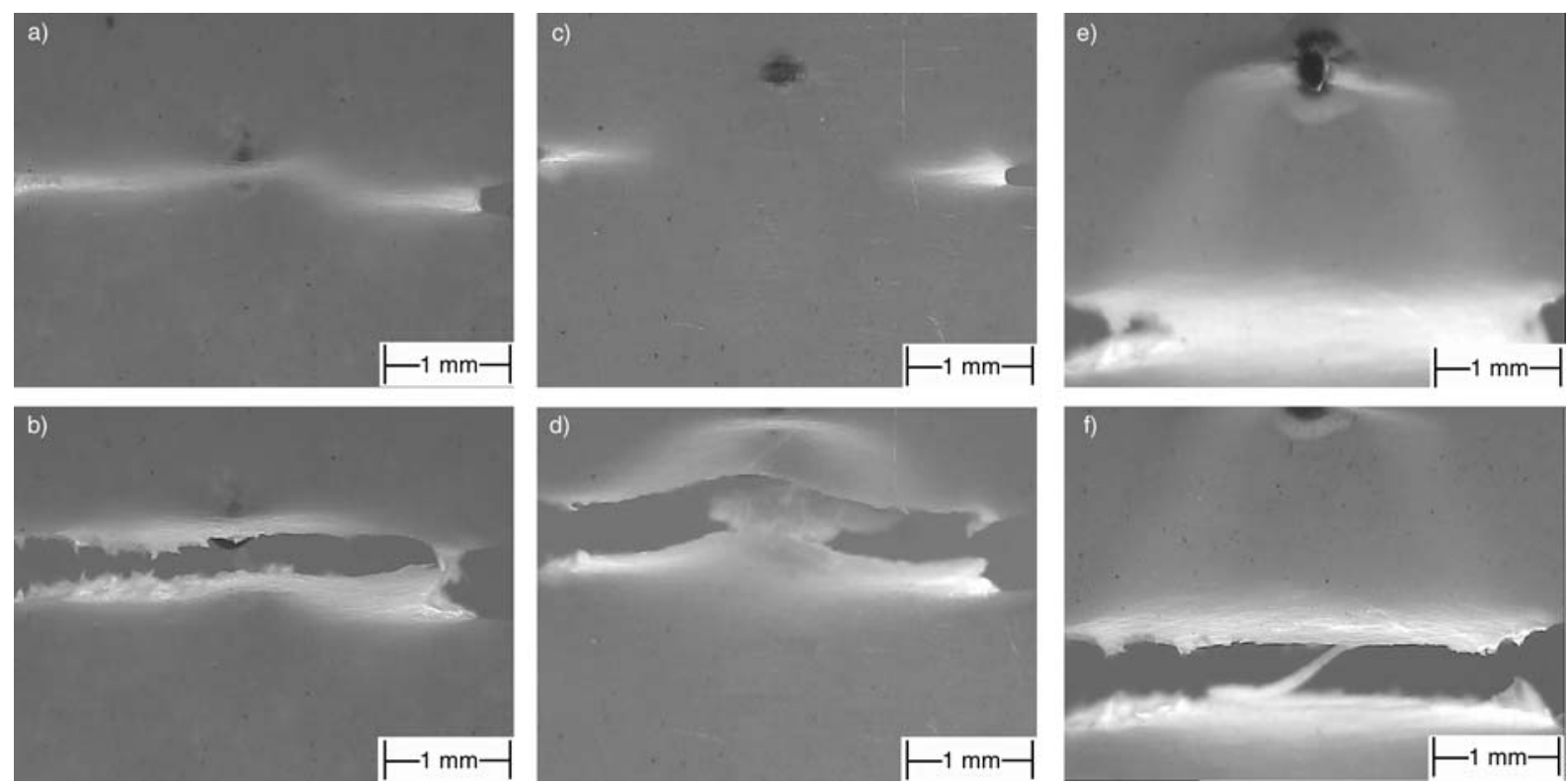

Figure 12. The effect of the distance of fiber heads from the theoretical failure cross section: the fiber head falls into the failure cross section (a) (b), the line of crack propagation inclines in the direction of the fiber head (c) (d), the fiber head has no significant influence on crack propagation (e) (f) 


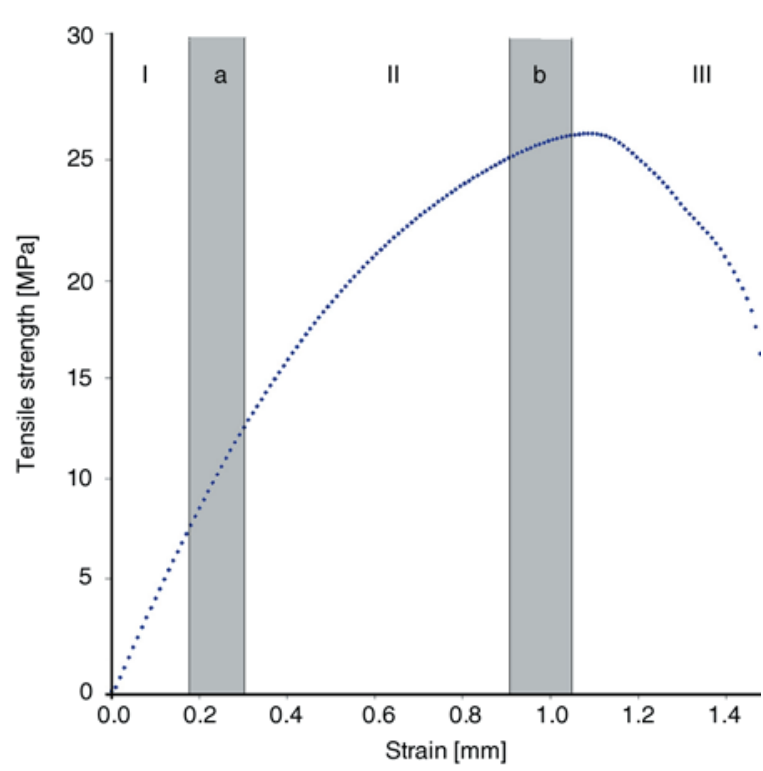

Figure 13. Characteristic single fiber head PP tensile curve: I the fiber and the matrix are deformed simultaneously, II yielding around the fiber head, III propagation of cracks initiating from the fiber head, a complete separation of the interface between the fiber head and the matrix, ceasing of adhesion, $b$ crack formation perpendicular to the direction of loading on the meridian of fiber heads

latter value is divided by the former one, an effective flaw area (assumed to be circular), i.e. a decrease in the cross section, was calculated. Based on the ratio of this circle and the radius of the fiber head, an effective sphere radius could be calculated. A characteristic tensile curve and its stages are illustrated in Figure 13.

Based on the aforementioned facts the process of the tensile test was as follows. In stage $I$ no change occurred in the structure of the sample, the deformation was totally reversible. In zone a the interfacial separation of the fiber head and the matrix occurred, and in stage II it was surrounded by an increasing yield vicinity (characteristically spherical) (Figure 12e). In zone b cracks formed on the meridian of the fiber head - depending on the distance from the theoretical tensile cross section and at the same time the cracks initiated from the notches, and finally total failure occurred (Figures 12a and $\mathrm{b}$ ). If the propagation direction of cracks initiating from around the fiber head differed from those starting from the notches, then their size was limited by the failure of the cross section (i.e. when loading stopped).

The influence of the distance of the fiber heads from the theoretical failure cross section on the ten-

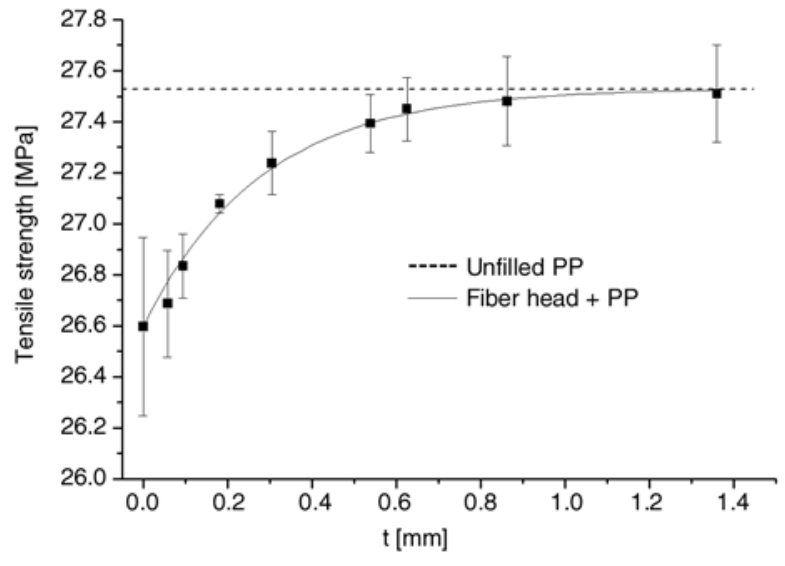

Figure 14. The influence of fiber heads on the tensile strength as a function of distance $t$ from the theoretical failure cross section

sile strength was analyzed during the above mentioned investigations (Figure 14).

A dotted horizontal line shows the tensile strength of the matrix in Figure 14. The measurement points approach the strength of pure PP (containing no additives) asymptotically as distance $t$ increases. An exponential curve was fitted on the points, and its general equation is the following (31):

$\sigma_{c}=\sigma_{c 0}+A_{1}\left(1-e^{-\frac{t}{t_{1}}}\right)$

at $R=0.990$ correlation factor, where: $\sigma_{c 0}=$ $26.60 \mathrm{MPa}, A_{1}=0.934 \mathrm{MPa}, t_{1}=0.280 \mathrm{~mm}$, and $t_{1}$ is the constant of the exponential part.

Based on the above mentioned facts it could be stated that if the distance between the fracture cross section and the fiber head is larger than (34) $\cdot t_{1}=0.84 \ldots 1.12$, the fiber heads had no significant influence on the measured strength. The shape of the curve corresponds to the assumptions of the theoretical model ( $r$ radius vicinity of $B$ ). In case of $0.5 \mathrm{~mm}$ distance, the decrease in strength occurred more definitely. In case of 0 distance, the decrease in strength reached $0.92 \mathrm{MPa}(3.34 \%)$. If the asymptotic character of the curve is neglected, and linear extrapolation is calculated from the first seven measurement points, then this influence ceases at $t=0.62 \mathrm{~mm}$. For the sake of simplicity, this range was considered to be the $\mathrm{r}$ radius vicinity of cross section $B$ in the model $\left(r=t_{0}=0.62 \mathrm{~mm}\right)$. Accordingly, within this range the strength was handled as an average constant, as the half of the total difference $(\Delta \sigma)(32)$ : 
$\Delta \sigma=\sigma_{m}-\sigma_{f h}$

where $\sigma_{m}$ is the tensile strength of the PP without fiber heads, $\sigma_{f h}$ is the tensile strength of PP containing one fiber head - in the effective vicinity of the tensile cross section.

Hence, if the effective spheres are handled as $100 \%$ defect sites, and are assumed to be exactly symmetrical to the plane of the failure cross section, $A_{\text {eff }}$ introduced in (23) can be determined with the help of (33), (34) and (35). From $A_{\text {eff }}$ the area of the meridian of the effective sphere $\left(A_{f h}\right)$ can be determined simply based on (36).

$\sigma_{m}=\frac{F_{0}}{A_{0}}$

$\sigma_{f h}=\frac{F_{f h}}{A_{0}}$

$\sigma_{m}=\frac{F_{f h}}{A_{e f f}}$

$A_{\text {eff }}=A_{0}-A_{f h}$

where $F_{0}$ is the tensile strength of $\mathrm{PP}$ without fiber head, $F_{f h}$ is the tensile strength of PP with one fiber head, while $A_{0}$ is the area of the failure cross section.

Knowing $A_{f h}$, the diameter $(d)$ of the - supposedly circular - area can be determined:

$d=\sqrt{\frac{4 A_{f h}}{\pi}}$

Knowing the diameter of the fiber heads used in the measurements, the value of $a_{e}$ introduced in (8) can be determined (38) with the help of (37).

$a_{e}=\frac{d}{d_{\text {fiber head }}}$

Based on Figure 14, $\sigma_{m}=27.52 \mathrm{MPa}$ and $\Delta \sigma=$ $0.46 \mathrm{MPa}$, and considering that during the measurement $A_{0}=4.88 \mathrm{~mm}^{2}, d_{\text {fiber head }}=223 \mu \mathrm{m}$, the ratio between the diameter of the effective sphere and that of the equivalent spheres turned out to be $a_{e}=1.81$.

Figure 15 reveals the influence of fiber head volume on the tensile strength.

The relation of fiber head volume and tensile strength is linear and can also be characterized by the general formula of (39):

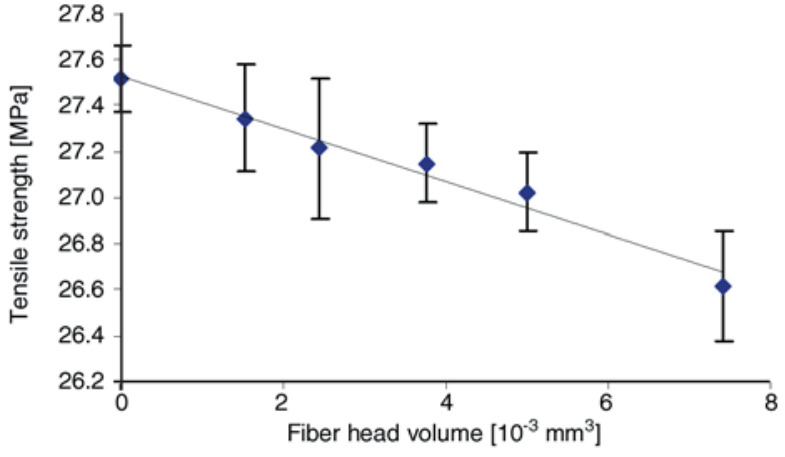

Figure 15. The influence of fiber head volume on the tensile strength

$\sigma_{c}=a_{c} V_{f h}+b_{c}$

at $R=0.986$ correlation, where $V_{f h}$ is the fiber head volume, $\quad a_{c}=-11.6 \cdot 10^{-3} \mathrm{MPa} / \mathrm{mm}^{3}$ and $b_{c}=$ 27.54 MPa. Furthermore, the linear relation verifies the correctness of the method of considering the effective spheres based on the ratio of their volume (Equation (21)).

\subsection{Application of the model}

The test results obtained on composites produced by melt mixing were supplemented by measurements carried out on specimens produced in the same way but using basalt fibers containing fiber heads. Figure 16 shows the tensile strength of composites with and without fiber heads as a function of fiber content. A polynomial curve of the fifth order was fitted on the measurement points showed in Figure 16 [12]. Differences appear between the strength values in the fiber content interval between 8.5 and $20 \mathrm{vol} \%$. In case of lower fiber contents, the effect of fiber head presence or absence is negligible. If the fiber content exceeds the upper value of the interval, the role of fiber heads is again less significant due to the discontinuities in the composite structure (the matrix does not fill the voids

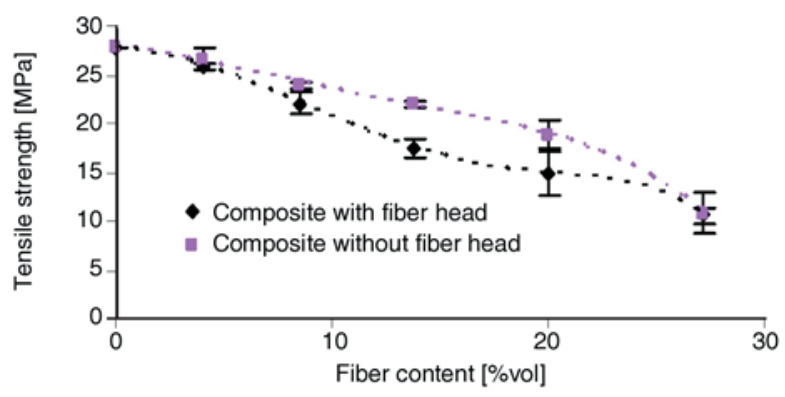

Figure 16. The influence of fiber heads on the tensile strength as a function of fiber content 
among the fibers sufficiently). Hence the model was examined only in the middle range $(8.5-20 \mathrm{vol} \%)$. The expected values of the composites containing fiber heads were calculated from the strength values of the coresponding material without fiber heads using the proposed model. The most important variable of the model is $a_{e}$, determined by finite element modeling and by investigating single fiber head composites. The aim of the finite element modeling was to obtain information on the size of the stress collection zones. If the stress collection zones exceeding the $0.92 \mathrm{MPa}$ difference relative to the far vicinity of the fiber head are considered, $a_{e}=1.67$ was obtained.

The $\Phi_{B V}$ values calculated from these results based on (21) provide the extent of strength reduction directly. The $\Phi_{B V}$ obtained in cases of different values (finite element modeling and tensile testing of PP specimens with single fiber head) are summarized in Table 4.

The results revealed in Table 4 are more illustrative in graphics. Hence the values measured in tensile tests on basalt fiber associated composites with and without fiber heads (Figure 16) and the results calculated based on the models are shown together in Figure 17.

Linear approximation was applied in the described stage. The uppermost line corresponds to composites without fiber heads, and the lowest one to the measured tensile strength of composites containing fiber heads. The line in the middle represents the calculations with the parameter values determined by finite element modeling. In this case the extent of strength reduction did not approach the real one.

Table 4. The values of $\Phi_{B V}$ as function of fiber content

\begin{tabular}{|l|c|c|c|}
\hline \multicolumn{1}{|c|}{ Fiber content $[\mathbf{v o l \%}$ ] } & $\mathbf{8 . 5}$ & $\mathbf{1 3 . 8}$ & $\mathbf{2 0 . 0}$ \\
\hline$\Phi_{B V}$ finite element $\left(a_{e}=1.67\right)$ & 0.942 & 0.907 & 0.865 \\
\hline$\Phi_{B V}$ tensile $\left(a_{e}=1.81\right)$ & 0.898 & 0.836 & 0.763 \\
\hline
\end{tabular}

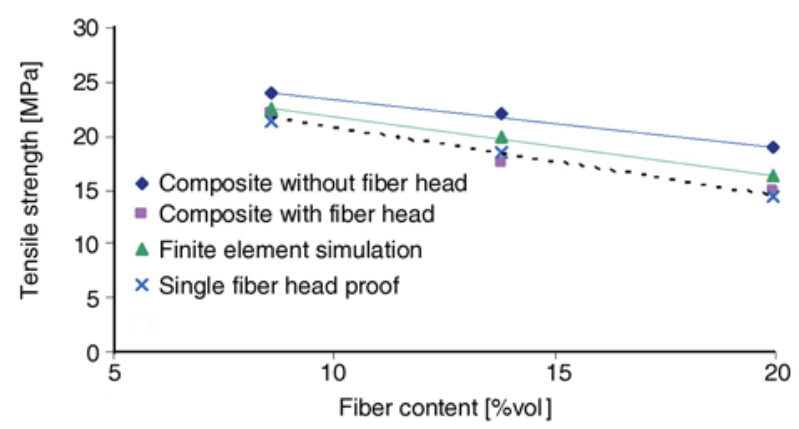

Figure 17. Comparison of the model and measured tensile strength values as a function fiber content
The reason is that although the model was developed further, in addition to interfacial processes relaxation phenomena could also take place, hence further refinement may be necessary to describe processes accurately.

The results obtained from single fiber head measurements correspond to the measurements carried out on composites with fiber heads. This is probably accidental, since the strength values of the composites with and without fiber heads deviate around the regression line in both directions and to different extents. Considering all the statements made, it was found that the model approximates the real processes well using parameter ae, hence the initial conditions and the principle turned out to be correct.

\section{Conclusions}

The influence of fiber heads, formed during the production of basalt fibers was studied in this article on the mechanical properties of composites. The fiber heads are of large size and number due to the characteristics of the technology. From the two main aspects related to the presence of these inclusions in the matrix, that is the improvement of toughness and defect site formation, the latter one turned out to be dominant. Firstly, the problem was analyzed by finite element simulations, in order to determine the intrinsic failure mode of the material. Afterwards, a theoretical model was created in characterizing the fiber head by an equivalent sphere, while its surroundings, the stress collection zone were described by another concentric sphere called effective sphere. The distribution of these effective spheres was described by a Poisson model. The main parameters of the model were determined with tensile tests on composites with a single fiber head embedded in PP. During these tests the influence of its size and the distance from the theoretical failure cross section on the tensile strength was analyzed. This way the effective cross section reduction, hence the role of fiber heads as volumetric defect sites in reducing the tensile strength were verified. The correctness of the model was verified by tensile tests made on composites made of basalt fibers with and without fiber heads. 


\section{Acknowledgements}

This work was supported by the Hungarian Scientific Research Fund (T049069 and NI62729) and the HungarianUkrainian Intergovernmental Science and Technology Program (TéT UKR-9/04). T. Czigány thanks the Öveges József Scholarship for the support of his personal research.

\section{References}

[1] Goldsworthy W. B.: New basalt fiber increases composite potential. Composites Technology, 8, 15 (2000).

[2] Kurkov V., Labok V.: Fibres from stone. International Textile Bulletin, 49, 48-52 (2003).

[3] Czigány T.: Basalt fiber reinforced hybrid polymer composites. Materials Science Forum, 473-474, 59-66 (2005).

[4] Gur'ev V. V., Neproshin E. I., Mostovoi G. E.: The effect of basalt fibre production technology on mechanical properties of fibre. Glass and Ceramics, 58, 24-27 (2001).

[5] Militky J., Kovacic V.: Influence of thermal treatment on tensile failure of basalt fibers. Engineering Fracture Mechanics, 69, 1025-1033 (2002).

[6] Czigány T.: Special manufacturing and characteristics of basalt fiber reinforced polypropylene composites: Mechanical properties and acoustic emission study. Composites Science and Technology, 66, 3210-3220 (2006).

[7] Czigány T., Pölöskei K., Karger-Kocsis J.: Fracture and failure behavior of basalt fiber mat-reinforced vinylester/epoxy hybrid resins as a function of resin composition and fiber surface treatment. Journal of Materials Science, 40, 5609-5618 (2005).
[8] Thomason J. L.: The influence of fiber length and concentration on the properties of glass fiber reinforced polypropylene: 5. Injection moulded long and short fiber PP. Composites Part A, 33, 1641-1652 (2002).

[9] Ségard E., Benmedahene S., Liksimi A., Lai D.: Influence of the fiber-matrix interface on the behaviour of polypropylene reinforced by short glass fiber above glass transition temperature. Composites Science and Technology, 62, 2029-2036 (2002).

[10] Matkó Sz., Anna P., Marosi Gy., Szép A., Keszei S., Czigány T., Pölöskei K.: Use of reactive surfactants in basalt fiber reinforced polypropylene composites. Macromolecular Symposia, 202, 255-267 (2003).

[11] Dmininghaus H.: Die Kunststoffe und ihre Eigenschaften. Springer-Verlag, Berlin (1998).

[12] Vas L. M., Balogh K.: Testing fiber orientation and its effect on glass mats by using image processing system. in 'Proceeding of VI. International Conference IMTEX'2000. Lodz, Poland', 69-78 (2000).

[13] Karlin S., Taylor H. M.: Stochastic Processes (in Hungarian). Gondolat Publisher, Budapest (1985).

[14] Vas L. M.: Statistical modeling of unidirectional fibrous structures. Macromolecular Symposia, 239, 159-175 (2006).

[15] Vas L. M.: Strength of unidirectional short fibre structures as a function of fibre length. Journal of Composite Materials, 40, 1695-1734 (2006).

[16] Vas L. M., Czigány T.: Strength modeling of twocomponent hybrid fiber composites in case of simultaneous fiber failures. Journal of Composite Materials, 40, 1735-1762 (2006).

[17] Sachs L.: Applied statistics. Springer-Verlag, Berlin (1978).

[18] Szabó J. S., Czigány T.: Static fracture and failure behavior of aligned discontinuous mineral fiber reinforced polypropylene composites, Polymer Testing, 22, 711-719 (2003). 